\title{
Dynamique des pratiques pastorales et des paysages : une approche pluri-échelles appliquée aux Pyrénées ariégeoises (France)
}

\author{
F Di Pietro ${ }^{1 *}$, G Balent $^{2}$ \\ ' JRC-ISIS, TP 650, I-2 I020 Ispra (VA), Italie ; \\ 2 Inra-Sad Toulouse, BP 27, F-31326 Castanet-Tolosan cedex France
}

(Reçu le 20 juin 1995; accepté le 14 février 1997)

\begin{abstract}
Summary - Dynamics of pastoral practices and landscape patterns: a multi-level approach in Pyrénées Ariégeoises (France). The coevolution of farming activities and landscapes in a village of Pyrénées Centrales is studied using an ecological indicator observed at the field level: grassland vegetation. Used within a model of vegetation dynamics under agricultural constraints, grassland vegetation is analysed at different levels of organisation of farming activities (the village and the farm) and of ecological processes (traditional and emergent landscape units). Grassland heterogeneity is studied at each level of organisation and has then been linked to the farming systems and land uses. At the farm level grassland heterogeneity is considered as an indicator of control of the farm's resources. At the level of the traditional landscape units ('terroirs') grassland heterogeneity is considered to be the result of three strategies of farmers towards the pastoral resource. An increased heterogeneity of grassland vegetation is observed and new broader landscape units are emergent. The scale of the landscape heterogeneity is changing according to a pattern presented in this paper. The use of an ecological indicator observed at the field scale allows us to link analysis of pastoral resource management and landscape dynamics, since the field is a level of organisation shared by both the agricultural and ecological processes.
\end{abstract}

landscape analysis / land use / biological indicator / mountain / hierarchy

Résumé - La dynamique des activités agricoles et des paysages dans une commune des Pyrénées centrales est étudiée en utilisant un indicateur écologique observé au niveau de la parcelle : la composition floristique des prairies, interprétée grâce à un modèle de dynamique de la végétation sous contrainte agricole. À partir des observations réalisées à l'échelle de la parcelle, des analyses sont conduites à différents niveaux d'organisation des activités agricoles (commune, exploitation agricole) et des processus écologiques (terroir, nouvelle unité paysagère). L'hétérogénéité de la flore prairiale à chacun de ces niveaux d'organisation est analysée et mise en relation avec des éléments du fonctionnement des exploitations et de l'utilisation du sol. L'hétérogénéité de la végétation au sein des exploitations agricoles est interprétée comme un degré de maîtrise des ressources prairiales. L'hétérogénéité de la végétation au sein des anciennes unités paysagères, des terroirs, est interprétée comme le résultat de la mise en ouvre, de la part des exploitants, de trois différentes stratégies par rapport aux ressources pastorales. L'hétérogénéité de la végétation prairiale au sein des terroirs augmente et de nouvelles unités paysagères, plus vastes que les terroirs, sont en émergence. L'hété-

* Correspondance et tirés à part.

Tél. : (39) 332785246 ; fax : (39) 332789394 ; courriel : francesca.di-pietro@jrc.it 
rogénéité du paysage change d'échelle et semble évoluer selon un modèle présenté ici. L'utilisation d'un indicateur écologique observé au niveau de la parcelle permet d'articuler l'analyse de la gestion des ressources pastorales et celle de la dynamique du paysage, car la parcelle constitue une échelle d'observation commune aux processus agricoles et écologiques.

analyse du paysage / utilisation de l'espace / indicateur biologique / montagne / hiérarchie

\section{INTRODUCTION}

L'émergence de problèmes d'environnement liés aux activités agricoles souligne l'importance du rôle de ces dernières dans la gestion des ressources naturelles. Cependant l'analyse des relations entre agriculture et environnement se trouve confrontée à la diversité des niveaux d'organisation pertinents pour les activités agricoles, d'une part, et pour les processus écologiques, d'autre part. Pour ce qui est des activités agricoles les niveaux d'organisation traditionnels sont en général facilement repérables. Qu'il s'agisse de la parcelle, de l'exploitation agricole, de la commune, de la petite région agricole, il existe un ensemble de découpages spatiaux qui permettent de rendre compte de ces activités (Deffontaines, 1973). Le problème est plus complexe dès qu'il s'agit de processus écologiques car, quelles que soient les questions abordées (flux de nitrates, érosion, biodiversité,...), les unités d'observation et d'analyse à prendre en compte ne sont pas aisément décelables (Allen, 1987 ; Burel et al, 1992). Ces niveaux d'organisation ne se superposent pas directement aux niveaux d'organisation classiquement utilisés pour décrire les activités agricoles. L'analyse des relations agriculture-environnement nécessite donc la définition d'une gamme d'échelles d'observation qui permettent d'articuler les processus écologiques et les activités agricoles et qui soient donc pertinentes pour ces deux types de processus (Meentemeyer et Box, 1987; Golley, 1989). À cette fin nous nous appuyons sur quelques principes issus de l'écologie systémique et plus particulièrement de la théorie de la hiérarchie (Allen et Starr, 1982).

i) Le territoire étant le lieu où se réalise l'articulation entre activités agricoles et processus écologiques, nous proposons d'étudier ces relations sur une portion continue de territoire, vu comme un ensemble de niveaux d'organisation hiérarchisés.

ii) Au sein de ce territoire nous définissons des unités paysagères, décelables sur les photographies aériennes et caractérisées par une homogénéité de la végétation à une certaine échelle. iii) L'analyse des interactions agriculture-environnement que nous présentons ici est basée sur l'utilisation de la parcelle comme échelle d'observation commune aux activités agricoles et aux processus écologiques. La parcelle est en effet la plus petite portion d'espace qui subit des pratiques agricoles homogènes; c'est à ce niveau que sont mis en œuvre des itinéraires techniques qui contrôlent de nombreux processus écologiques (dynamique de la végétation, flux de nutriments, érosion...) ; la parcelle constitue donc une échelle pertinente pour rendre compte du fonctionnement de ces processus.

Nous considérons la parcelle comme la plus petite portion d'espace commune aussi bien aux activités agricoles qu'aux processus écologiques (fig 1). Il s'agit de l'unité de résolution de l'observation («grain » : Allen et al, 1984 ; Wiens, 1989 ; Baudry, 1990) à partir de laquelle nous allons reconstituer, par agrégation, les unités d'analyse supérieures pertinentes pour chaque processus: les exploitations agricoles (activités agricoles) et les terroirs (processus écologiques).

Notre objectif dans cet article est de montrer l'intérêt d'une démarche hiérarchique pour la définition d'unités d'analyses pertinentes à l'étude des interactions agriculture-environnement.

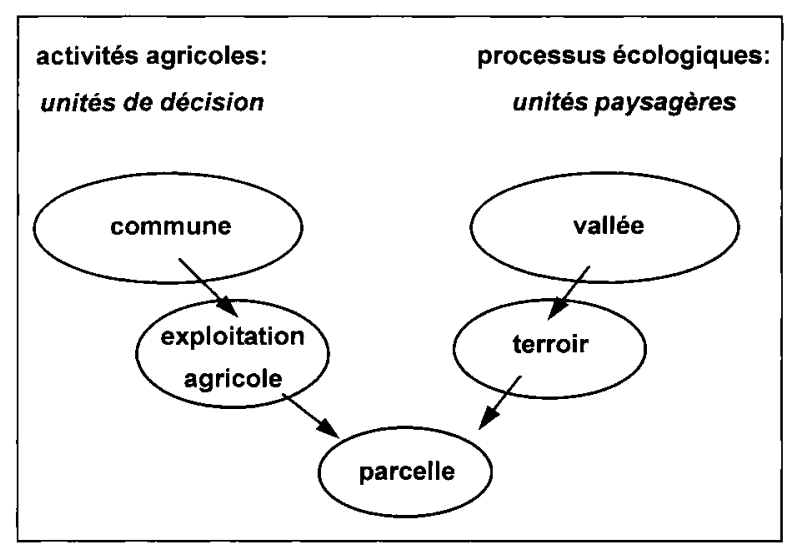

Fig 1. La parcelle, un niveau d'organisation commun aux activités agricoles et aux processus écologiques (les niveaux d'organisation représentés sont valables dans les Pyrénées centrales). 


\section{MATÉRIEL ET MÉTHODE}

\section{Le système pastoral des Pyrénées centrales}

Les systèmes pastoraux pyrénéens servent de support pour illustrer notre approche. Ces systèmes présentent plusieurs avantages pour l'analyse des relations agriculture-environnement. L'utilisation du territoire y est basée sur l'exploitation d'une ressource polymorphe mais unique : la prairie permanente. Les relations entre pratiques agricoles et territoire sont donc plus simples à analyser que dans le cas des régions de polycultureélevage. Cette ressource, objet de gestion, est aussi le principal élément constitutif des paysages de vallée; ceci fait de la végétation prairiale un indicateur biologique privilégié pour l'analyse des dynamiques agricoles et écologiques. Dans ce cas l'hétérogénéité du paysage est en grande partie due à l'hétérogénéité de la végétation prairiale.

\section{Modélisation du système et du paysage pastoral pyrénéen}

Les interactions herbivores-végétation ont été modélisées dans le cadre du système pastoral, considéré comme un système écologique piloté par l'homme (Balent et Gibon, 1988 ; Balent, 1989). Son fonctionnement, jusqu'à une période récente, peut être vu comme l'articulation de trois niveaux d'organisation hiérarchiques, caractérisés par des échelles spatio-temporelles spécifiques : la commune, l'exploitation agricole, la parcelle (fig 2). Ce modèle permet de définir

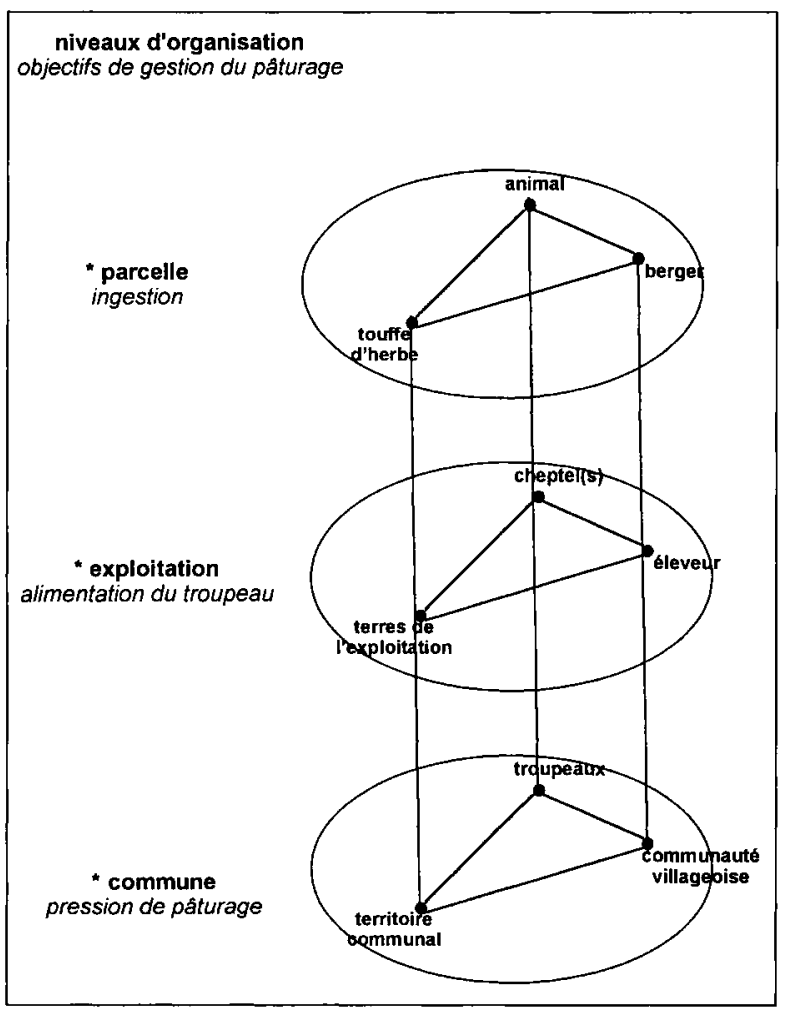

Fig 2. Hiérarchie de niveaux d'organisation agricoles : modélisation du système pastoral (d'après Balent, 1986a). des unités spatiales pertinentes en termes d'activités agricoles - la commune, les exploitations agricoles et leurs parcelles - et de systèmes écologiques - la vallée, les terroirs, les parcelles.

La commune représente, dans les Pyrénées, un important niveau de gestion des activités agricoles (Chevalier, 1956). À ce niveau le conseil municipal gère l'utilisation des différentes surfaces de vallée par les troupeaux présents sur la commune en réglementant le pâturage, sur un pas de temps pluriannuel, par des décisions qui limitent les pratiques agricoles pouvant être mises en œuvre par les exploitants. Celles-ci assurent ainsi la reproduction à long terme des ressources pastorales communales.

Au niveau de l'exploitation agricole, l'utilisation des surfaces de l'exploitation peut être vue comme l'ajustement, par l'agriculteur, des besoins alimentaires de son troupeau et des surfaces de son exploitation. Cet ajustement est réalisé par des décisions prises à l'échelle de la campagne annuelle.

Au niveau de la parcelle le berger gère l'utilisation de la végétation par les animaux et tout particulièrement l'ingestion au pâturage pendant la journée.

Cette organisation ancienne a façonné le paysage pastoral en terroirs. Les terroirs sont des portions d'espace continu ayant eu, jusqu'au milieu de ce siècle (Chevalier, 1956 ; confirmé dans les Alpes par Delcros, 1992), une homogénéité d'utilisation anthropique basée sur l'homogénéité des aptitudes agronomiques (Bertrand, 1975 ; Inra-Enssaa, 1977 ; Deffontaines, 1988). Ils sont donc le produit d'une utilisation maximale de l'espace disponible, dans le cadre d'une agriculture de subsistance; chaque terroir avait alors une fonction spécifique au sein du système de production et constituait pour les exploitations un type particulier de ressource: terroirs cultivés (cultures vivrières pour l'alimentation familiale et cultures fourragères pour certains cheptels), terroirs fauchés (constitution de stocks fourragers hivernaux), terroirs de parcours (pacages de demi-saison pour différents lots d'animaux). Cette utilisation particulière entraînait une intensité variable aussi bien des prélèvements que des apports de matière organique; les terroirs cultivés étaient alors privilégiés et fertilisés plus régulièrement que les prairies permanentes. Après l'abandon des cultures, le niveau de fertilité spécifique à chaque terroir a conservé une grande influence sur la dynamique de la végétation dans le cadre de pratiques agricoles évoluant très lentement (Balent et Duru, 1984). Les terroirs, définis par une fonction agricole, associent donc à l'homogénéité des aptitudes agronomiques et de l'utilisation anthropique passée une homogénéité de la dynamique végétale. Ceci en fait des unités paysagères traditionnelles des paysages pastoraux ; il s'agit d'unités fonctionnelles (des ressources liées à une fonction du système de production agricole) et structurelles (des unités paysagères) des systèmes agroécologiques de montagne.

Parmi les niveaux d'organisation du système pastoral, seules l'exploitation agricole et la parcelle jouent encore aujourd'hui un rôle clé ; le niveau d'organisation communal a été fortement affaibli par l'évolution 
de l'agriculture de montagne, laissant ainsi les exploitations agricoles individuelles maîtresses du choix des pratiques agricoles mises en cuvre (Balent et Stafford Smith, 1993). L'affaiblissement du niveau d'organisation communal et la diversification des exploitations expliquent l'actuelle déstructuration des paysages pastoraux et la perte de lisibilité des terroirs : aujourd'hui ceux-ci ne sont pas toujours associés à une fonction agricole spécifique; les parcelles les composant sont souvent utilisées de différentes façons. Les différences entre les terroirs s'estompent; à une échelle plus large émerge une nouvelle organisation spatiale du paysage pastoral, constituée de nouvelles unités paysagères plus vastes que les terroirs.

\section{La flore prairiale, indicateur de la gestion agricole des ressources du territoire}

Pour comparer les prairies permanentes des différents terroirs nous avons utilisé un modèle d'évolution de la flore prairiale qui réduit la diversité des prairies à deux facteurs de variation principaux : la fertilité résiduelle et l'intensité d'utilisation (Balent et Duru, 1984; Balent, 1986 ; 1991). Ce modèle traduit la dynamique d'évolution de la flore d'une parcelle entre le moment où la culture y est abandonnée et celui où elle se stabilise sous la forme d'une pelouse oligotrophe - au bout de 80 à 100 ans après l'abandon de la culture - ou sous la forme d'un taillis - une cinquantaine d'années après l'abandon de la culture, si la parcelle est située à proximité d'un bois ou d'une haie (fig 3). L'évolution de la flore prairiale est sous la double influence des pratiques agricoles et des facteurs du milieu; or ce modèle est valable lorsque le rôle des pratiques agricoles sur la dynamique de la végétation est prépondérant et la parcelle agricole représente un niveau d'analyse essentiel dans les relations entre activités agricoles et processus écologiques.

\section{La commune d'Ercé}

La commune d'Ercé (fig $4 a$, b) se trouve dans les Pyrénées ariégeoises, au sein du haut Couserans, une entité naturelle qui est aujourd'hui exclusivement tournée vers l'élevage, axé principalement sur la production de viande bovine; la prairie permanente y occupe plus de $95 \%$ de la superficie agricole utilisée (Sau).

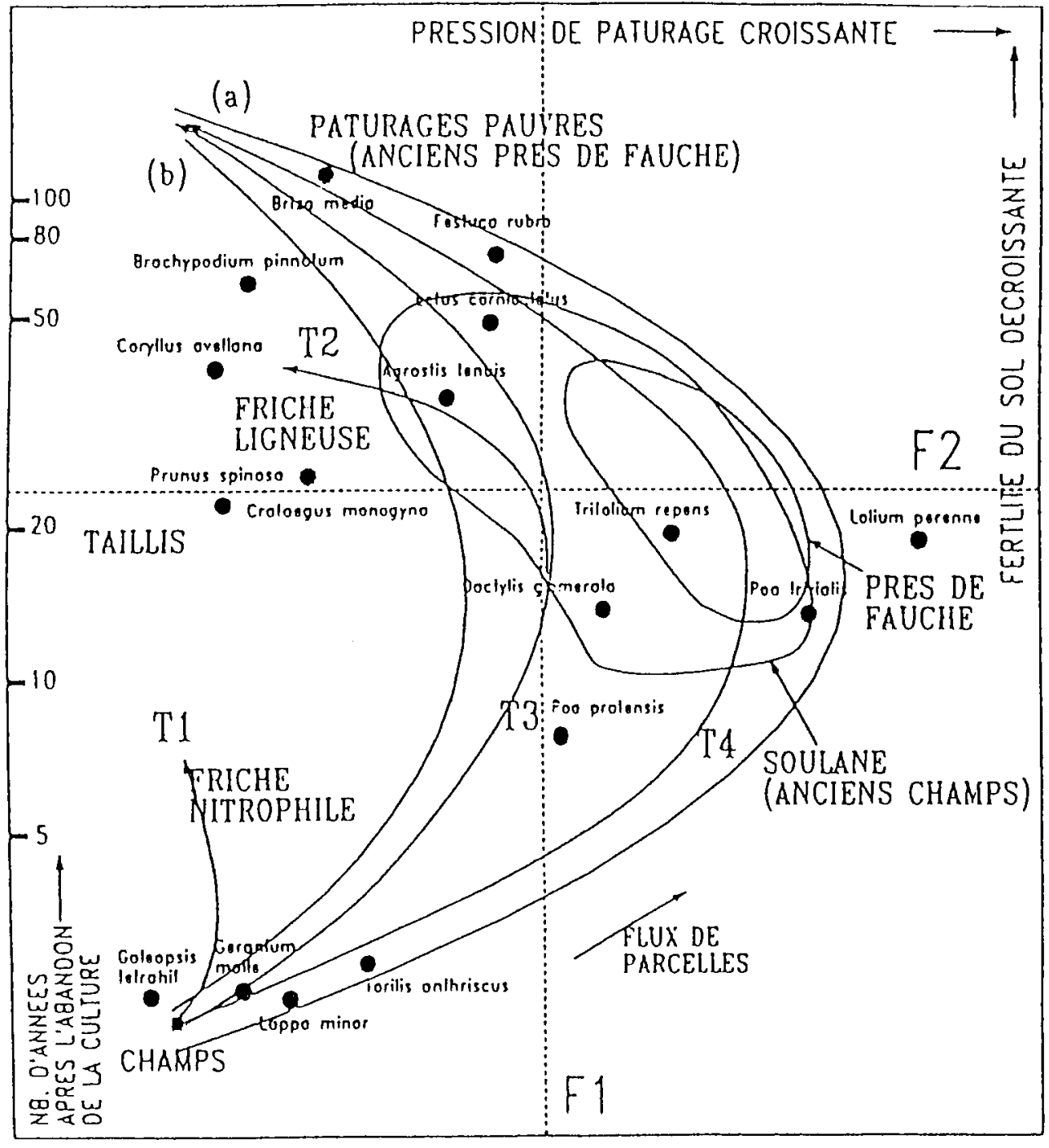

Fig 3. Le modèle de dynamique de la végétation (d'après Balent, 1986). 


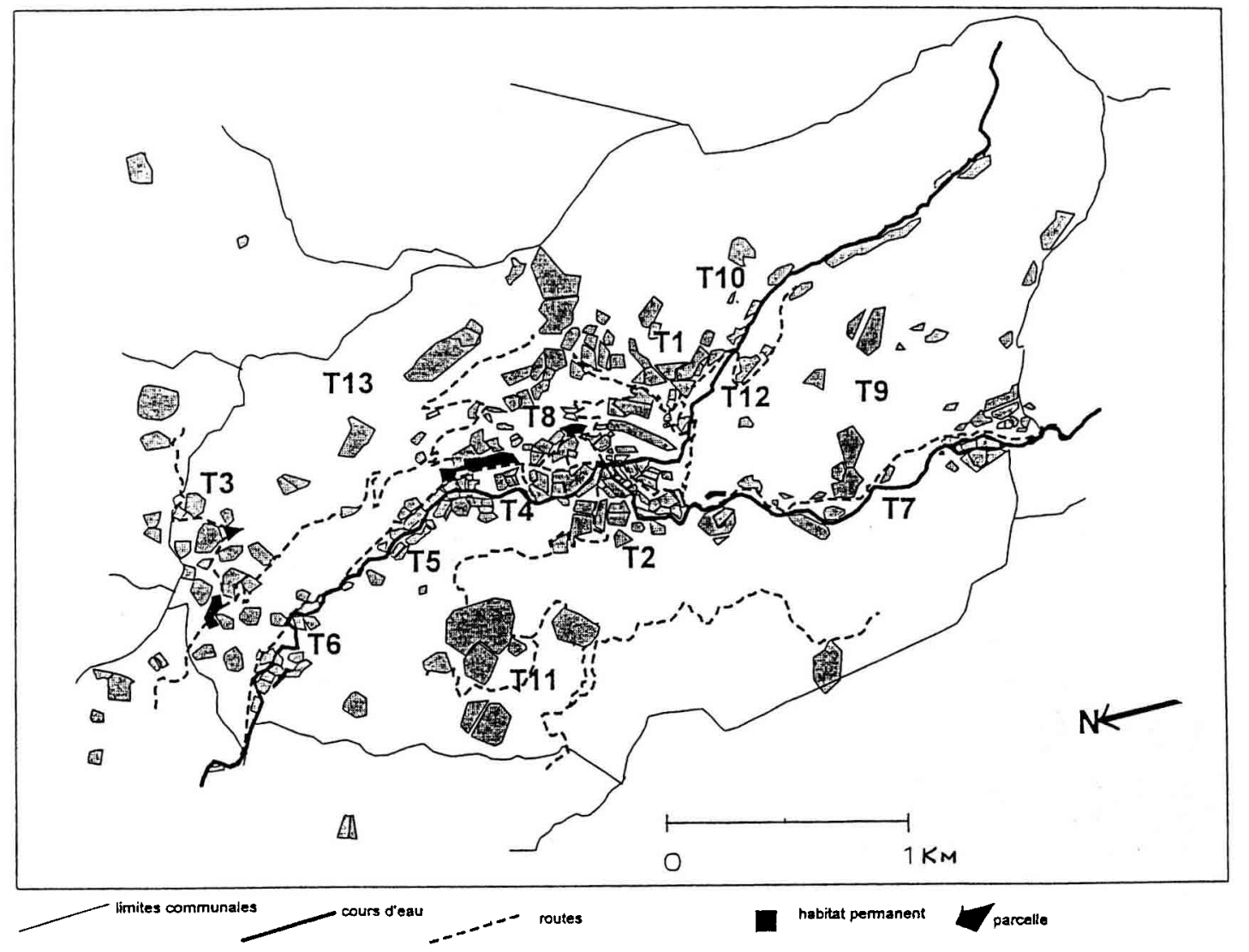

a.

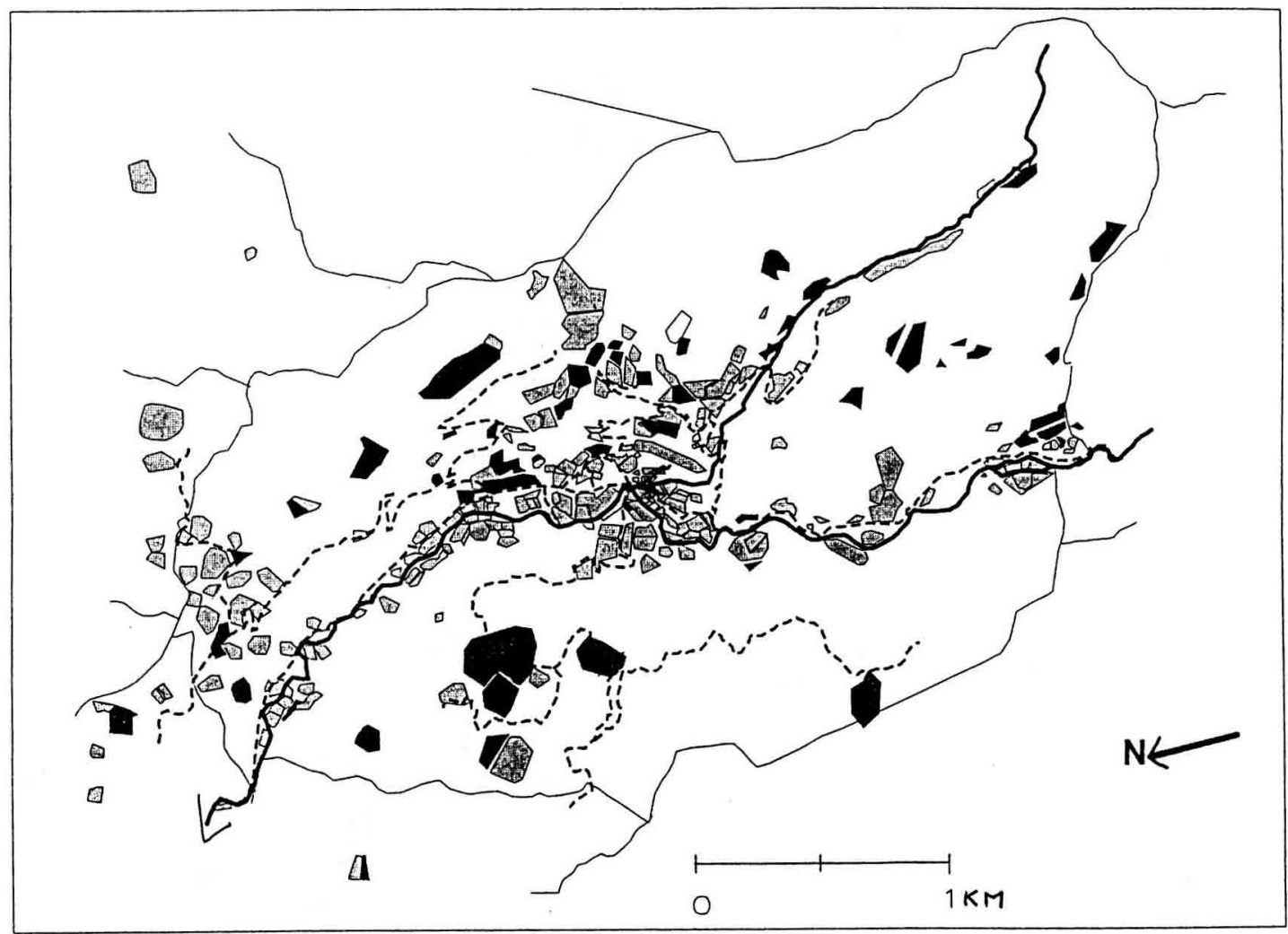

b.

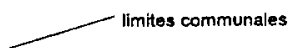

habitat permanent près de fauche

Fig 4a. Carte des terroirs d'Ercé. La carte montre l'appartenance des parcelles actuellement exploitées, représentées en grisé, aux différents terroirs ( $\mathrm{Ti}$ = numéro du terroir). Les surfaces en blanc sont boisées ou rocheuses. b. Carte de l'utilisation actuelle des parcelles de prairie permanente à Ercé (les prés de fauche figurent en gris clair, les pacages en gris foncé). 
Du point de vue agricole la commune d'Ercé est une des communes les plus dynamiques du haut Couserans : ses 4075 ha de Sau, situés entre 580 et $1300 \mathrm{~m}$ d'altitude, sont aujourd'hui utilisés par 28 exploitations agricoles. Le système d'élevage traditionnel repose sur l'utilisation d'une race locale rustique (vache gasconne) avec vêlages de printemps et vente de veaux broutards à l'automne, après la descente des pacages d'altitude (estives). Le troupeau est alimenté essentiellement à partir des surfaces de l'exploitation et des estives, utilisés durant la longue saison de pâturage d'été.

Le territoire d'Ercé, dont le village est situé à $650 \mathrm{~m}$ d'altitude, s'étend sur une vallée en auge avec un fond plat - très favorable à une conduite mécanisée des prairies principalement fauchées - et des versants très en pente, souvent en cours d'enfrichement, qui sont utilisés pour la fauche et pour le pâturage. Ces versants sont parsemés de «bordes», parcelles clôturées par une haie vive et associées à des granges-étables permettant de stocker le foin récolté sur place et distribué aux animaux en demi-saison en complément du pâturage (Viviani Rossi et al, 1992). Les contraintes techniques et les problèmes de main d'œuvre que connaît l'agriculture de montagne expliquent l'abandon de la fauche sur les versants et le changement progressif de la fonction des bordes : d'une utilisation en prés de fauche, le plus souvent pâturés en demi-saison, elles évoluent vers une utilisation en pacages, parcelles seulement pâturées, parfois en demi-saison, voire simplement en été. La fauche pour la constitution des stocks fourragers hivernaux est réalisée uniquement sur le fond de vallée, voire à l'extérieur de la zone de montagne, sur des terres louées ou achetées à cet effet (Gibon, 1981).

\section{Méthodes de recueil des données}

\section{Données sur les terroirs}

D'après la définition des terroirs mentionnée précédemment, nous avons identifié ceux de la commune d'Ercé par photo-interprétation des clichés photographiques IGN de 1942 et 1953 (utilisation du sol dans les années 1940 et 1950) et analyse de la carte topographique IGN à l'échelle $125000^{\mathrm{e}}$ (caractéristiques topographiques). Quinze terroirs ont été identifiés sur le territoire communal : cinq cultivés, neuf fauchés et un uniquement pâturé à cette époque-là (tableau I et fig 4a). Le fond de vallée était autrefois partagé entre quatre terroirs : deux situés en zone non inondable et cultivés (T4 et T5) et deux fauchés, trop humides pour les cultures (T6 et T7) ; les terrasses glaciaires hautes, non inondables et à faible pente, étaient cultivées. Deux versants exposés au sud (soulanes) constituaient les terroirs privilégiés des cultures (la soulane d'Ercé et la soulane de Cominac, hameau d'Ercé). Les huit terroirs restants étaient des terroirs bocagers, situés sur les versants et constitués de bordes fauchées : les bordes proprement dites (deux terroirs, T7 et T9), les trois ombrées (versants exposés au nord), la soulane éloignée (terroir anciennement fauché car trop éloigné du village pour être cultivé), les deux terroirs situés sur les hauts versants : le terroir de «bordes hautes» (sur le versant exposé à l'ouest, autrefois utilisé en pacage de demi-saison lors de la transhumance d'été, quelquefois fauché aujourd'hui) et le plateau de Lasserre (exposé à l'est, autrefois utilisé pour la fauche et pour l'estivage des bovins, c'est aujourd'hui un terroir utilisé surtout dans le cadre d'associations foncières pastorales). Aujourd'hui la plupart de ces terroirs connaissent deux fonctions : prés de fauche, le plus souvent pâturés en demi-saison, et pacages (non fauchés). Seuls trois terroirs du fond de vallée (T5, T6, T7) et la soulane d'Ercé (T1) connaissent une seule fonction et sont constitués uniquement de prés de fauche (fig 4b).

\section{Données sur les pratiques agricoles}

Une enquête dans les 28 exploitations agricoles de la commune d'Ercé a été réalisée en 1992 (Di Pietro, 1996). L'utilisation, au cours de l'enquête, d'une photographie aérienne récente de la commune (mission IGN de 1983 ou de 1991), sur laquelle nous avons demandé aux éleveurs de dessiner le parcellaire de leur exploitation, a permis d'identifier 202 parcelles d'utilisation, disséminées dans le territoire de la commune. Un questionnaire a permis de définir, pour chacune, les opérations techniques mises en ouvre et les principales caractéristiques du milieu.

\section{Données sur la végétation}

Parmi les 202 parcelles identifiées, 174 parcelles présentaient des données exploitables. Nous en avons retenu 129 appartenant à 16 exploitations, situées sur 13 des 15 terroirs de la commune (fig 5), échantillonnées de façon à obtenir la plus grande diversité des opérations techniques mises en œuvre. Sur chacune de ces parcelles nous avons effectué un relevé botanique ; les relevés ont été réalisés avec la méthode dite des «pointsquadrats " (adaptée par Daget et Poissonnet, 1971), qui est la méthode servie à l'élaboration du modèle de dynamique de la végétation utilisé ; les espèces de chaque parcelle ont été relevées en présence/absence, dans 50 points situés le long d'une ligne de $20 \mathrm{~m}$, avec un écartement régulier de $40 \mathrm{~cm}$ entre deux points. Les profils floristiques des parcelles ont été projetés en individus supplémentaires dans le modèle de dynamique de végétation. Chaque parcelle est ainsi caractérisée par deux abscisses, l'une sur l'axe de la fertilité résiduelle, l'autre sur l'axe d'intensité d'utilisation.

\section{RÉSULTATS}

\section{La gestion agricole des ressources du territoire}

\section{Au niveau communal}

Projeté dans le modèle de référence, notre échantillon de parcelles ne se distribue que dans la par- 


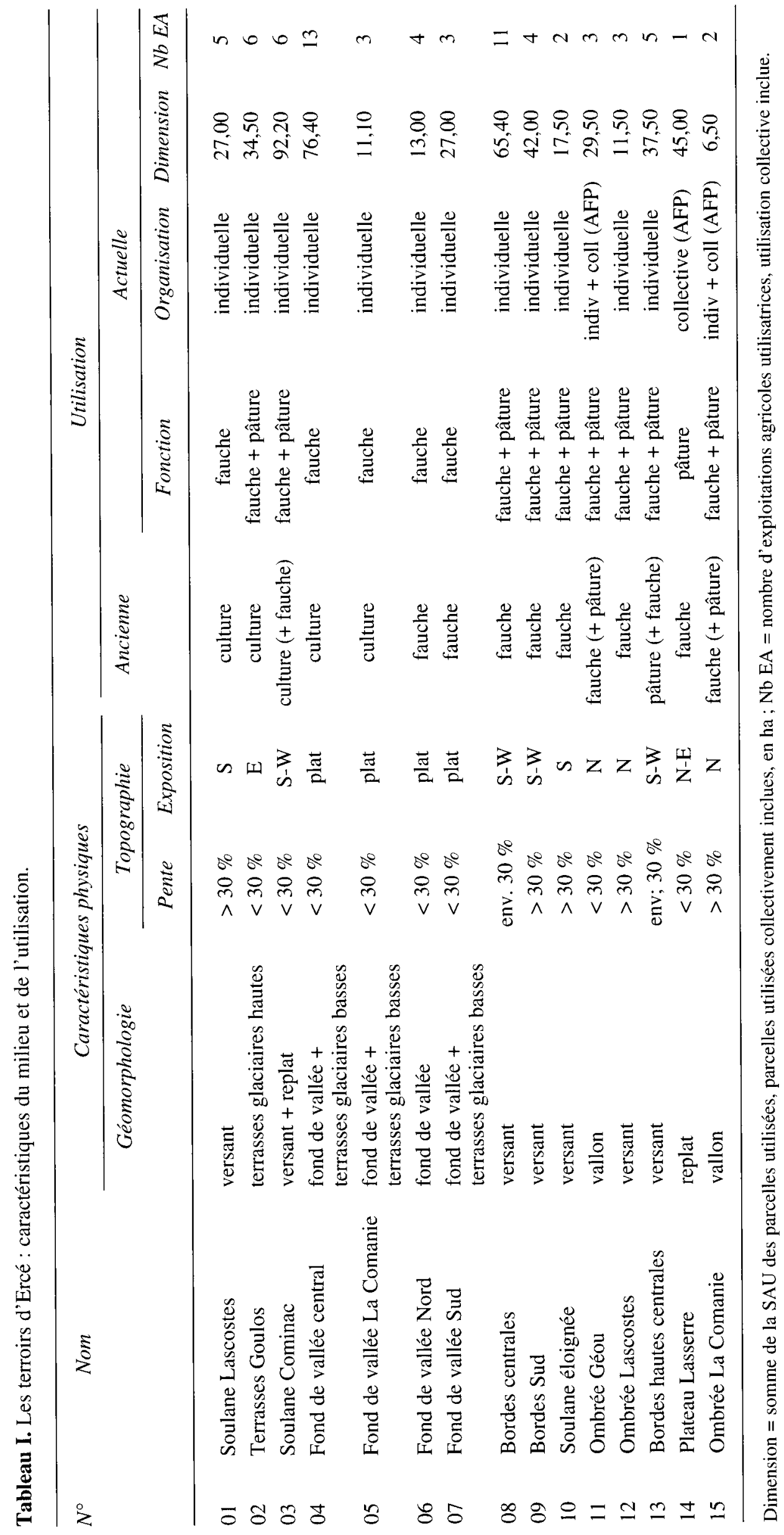


tie du modèle décrivant l'évolution de la végétation à partir du stade prairial (haut de la figure 3). La première partie du modèle, qui décrit la dyna-

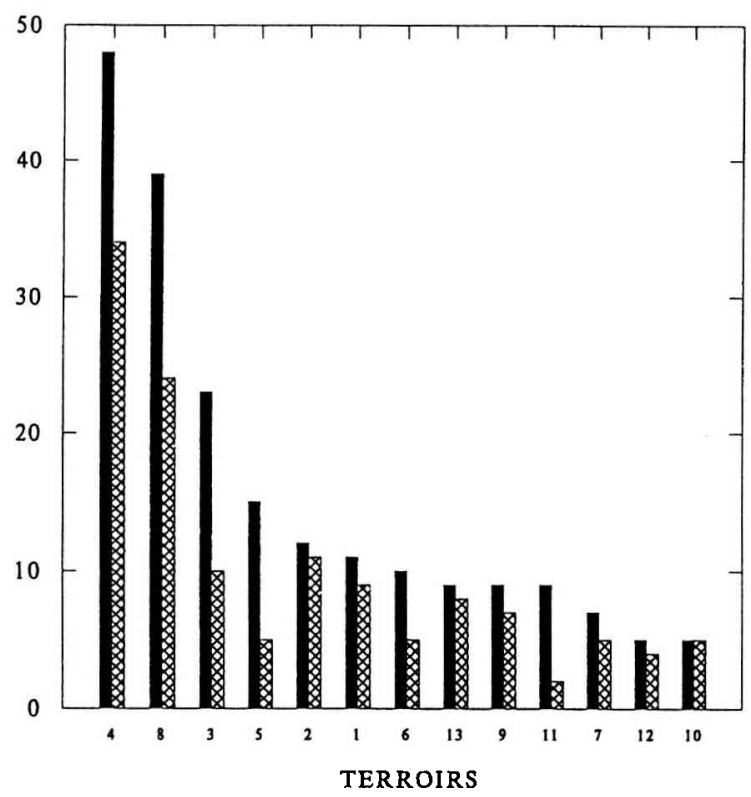

parcelies échantillonnées (total: 129)

m parcelles relevées en enquete (total: 202)

Fig 5. Ercé : échantillonnage des parcelles par terroir. mique de la végétation depuis l'abandon de la culture jusqu'à l'établissement d'une flore prairiale, environ 20 ans après l'abandon de la culture, n'est pas présente à Ercé, où la dernière culture a été effectivement abandonnée au cours des années 1960. La distribution relativement linéaire des parcelles dans le modèle témoigne d'une relation générale entre la fertilité et l'intensité d'utilisation des prairies et définit le patron de l'évolution de la végétation prairiale propre à cette commune (fig 6).

Cent trente espèces prairiales ont été recensées au total dans les prairies permanentes d'Ercé. Les prairies les plus fertiles et utilisées de façon très intensive (deux coupes de foin, pâturage de demisaison, fertilisation organique et minérale régulière) sont caractérisées par la dominance de Lolium perenne, Dactylis glomerata et Chaerophyllum aureum, alors que les prés de fauche moins utilisés et faiblement fertiles sont caractérisés par Rhinantus minor, Poa trivialis, Trifolium repens. Les pacages et prés de fauche avec une intensité d'utilisation moyenne et une fertilité faible et les pacages oligotrophes avec une intensité d'utilisation et une fertilité minérale très faibles sont caractérisés par des espèces telles que Festuca rubra, Potentilla erecta, Pimpinella saxifraga.

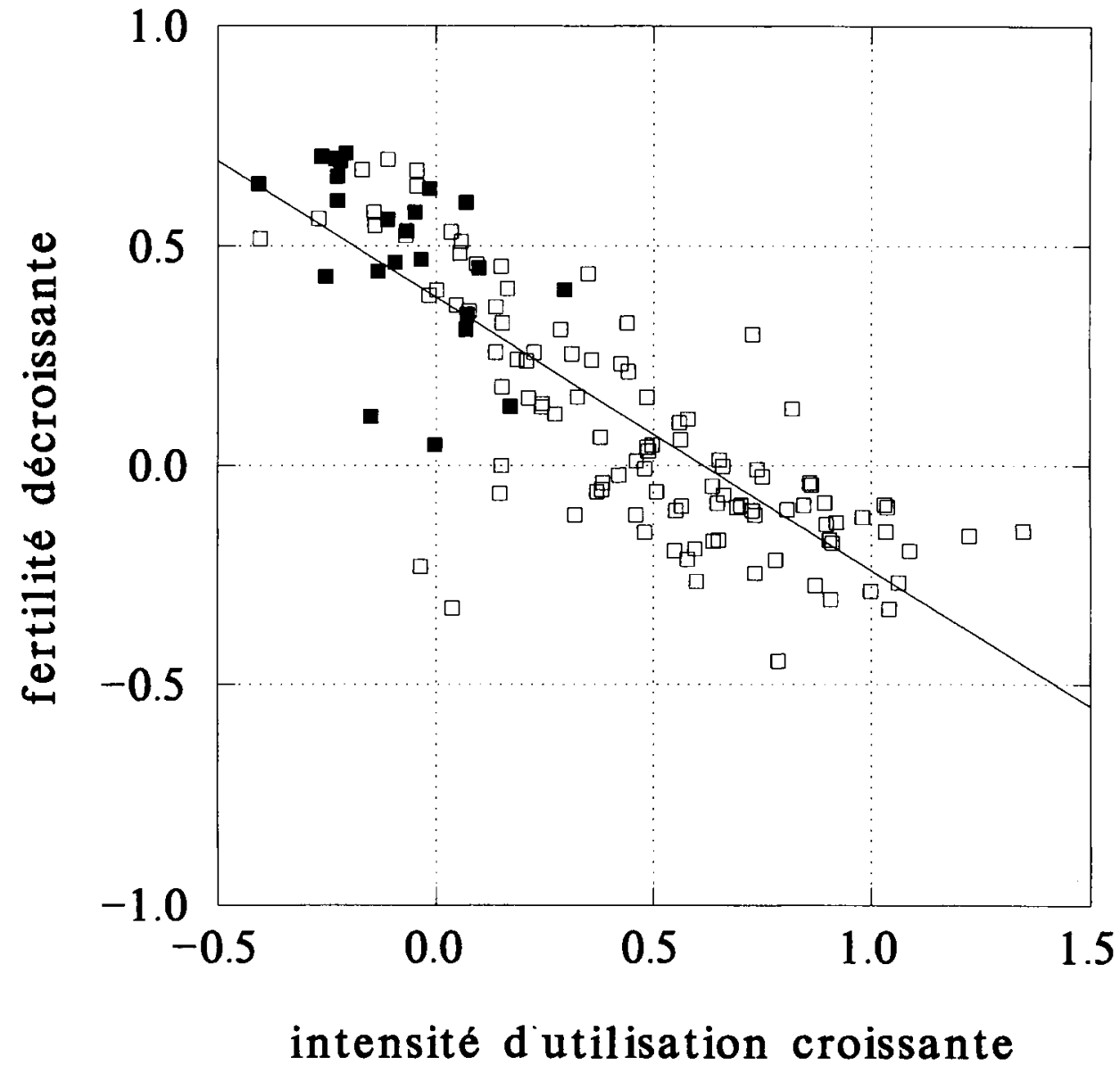

Fig 6. Dynamique de la végétation à Ercé (les carrés blancs représentent des parcelles utilisées en prés de fauche, les carrés noirs représentent des parcelles utilisées en pacage; pour les figures de 6 à $9 \mathrm{c}$ les axes sont gradués selon les coordonnées factorielles du modèle de dynamique de la végétation utilisé). 
Quelques parcelles s'éloignent de ce patron. Il s'agit d'une part de parcelles sous-utilisées par rapport à leur fertilité (friches nitrophiles), représentant les dernières parcelles de culture abandonnées dans la commune, caractérisées par Urtica dioica, Artemisia vulgaris et, d'autre part, de quelques parcelles sur-utilisées en regard de leur niveau de fertilité minérale.

\section{Au niveau des exploitations agricoles}

Sur les 16 exploitations agricoles, 13 exploitations (100 parcelles) ont un échantillonnage botanique sur au moins $80 \%$ de leur Sau. Au sein de ces 13 exploitations les relations fertilité-utilisation des prairies présentent une grande diversité : la localisation des parcelles sur la diagonale représentant la tendance dominante dans l'échantillon est en effet très variable suivant les exploitations. Nous pouvons distinguer trois types de configurations.

i) Un premier groupe de cinq exploitations présente une distribution des parcelles le long de cette diagonale (fig 7a). Ces exploitations ne comportent pas de parcelles sous-utilisées ou sur-utilisées par rapport à leur fertilité minérale ; l'intensité d'utilisation semble bien adaptée à la fertilité minérale et les ressources pastorales bien valorisées. Seul ce premier groupe d'exploitations semble ajuster l'utilisation des prairies et leur fertilité minérale, ce qui peut être interprété comme une bonne maîtrise dans la gestion des ressources pastorales.

ii) Un deuxième groupe de six exploitations présente la même configuration à l'exception de quelques parcelles où l'intensité d'utilisation n'est pas ajustée à la fertilité (fig 7b). Il s'agit de six exploitations où une ou deux parcelles sont sousutilisées par rapport à leur fertilité minérale et présentent une dynamique d'enfrichement.

iii) Un troisième groupe de deux exploitations montre des relations fertilité minérale-intensité d'utilisation tout à fait éloignées de la tendance générale d'évolution à Ercé et ne présente pas de patron clair (fig 7c); ici aussi nous trouvons des parcelles sous-utilisées par rapport à la fertilité minérale.

\section{Les effets des pratiques agricoles sur le paysage}

\section{À l'échelle des terroirs}

Au sein des 13 terroirs d'Ercé analysés, nous pouvons distinguer trois groupes de terroirs, en fonction de l'hétérogénéité de la végétation. i) Des terroirs avec une végétation homogène et caractérisée par des prairies utilisées et fertilisées intensivement (cinq terroirs; fig 8a). C'est le cas de l'ensemble du fond de vallée et des terrasses glaciaires, qui sont presque uniquement fauchés.

ii) Des terroirs avec une végétation homogène et caractérisée par un niveau d'utilisation et un niveau de fertilité bien plus faibles que sur les terroirs de fond de vallée (trois terroirs; fig $8 b$ ). Il s'agit de terroirs de versant difficilement mécanisables (pente supérieure à $30 \%$ ) : terroirs 10 , $12,14$.

iii) Des terroirs avec une végétation hétérogène (cinq terroirs ; fig 8c). C'est dans ce dernier groupe que l'extension le long de la diagonale représentant la tendance d'évolution à Ercé est maximale: ces terroirs présentent la plus forte hétérogénéité de la végétation et des pratiques. Il s'agit généralement de prairies assez pauvres, sur versant (terroirs 3, 8, 9, 13). Le terroir 1 (la soulane Lascostes) est au contraire un terroir encore fertilisé et utilisé (absence de pacages et utilisation exclusive en prés de fauche), malgré sa pente supérieure à $30 \%$; cela s'explique par son histoire culturale (terroir de culture bien fertilisé).

\section{À l'échelle des unités paysagères émergentes}

Trois unités paysagères peuvent aujourd'hui être identifiées à Ercé (source : photo-interprétation de clichés IGN de 1983 et de 1991) : une unité composée par l'ensemble du fond de vallée, et deux unités situées sur les versants: les soulanes (sur les versants exposées au sud) et les bordes (sur des versants à l'exposition variable). Le fond de vallée, fauché, constitue désormais une seule unité spatialement continue. Deux unités paysagères discontinues se trouvent sur les versants : les deux soulanes, composées de parcelles «ouvertes» (sans haie) car anciennement cultivées, sont aujourd'hui fauchées et pâturées ; les « bordes ", entourées de haies de moins en moins entretenues et tendant à un boisement continu, sont utilisées en prés de fauche et en pacages.

C'est seulement sur l'unité de fond de vallée que les niveaux fertilité minérale-intensité d'utilisation sont homogènes et se situent à un niveau élevé : les parcelles sont bien fertilisées et utilisées intensivement en prés de fauche (fig 9a). Sur les bordes, ces niveaux sont plus diversifiés et se situent en général à un niveau plus bas de fertilité (fig 9b), comme pour les soulanes (fig 9c). 


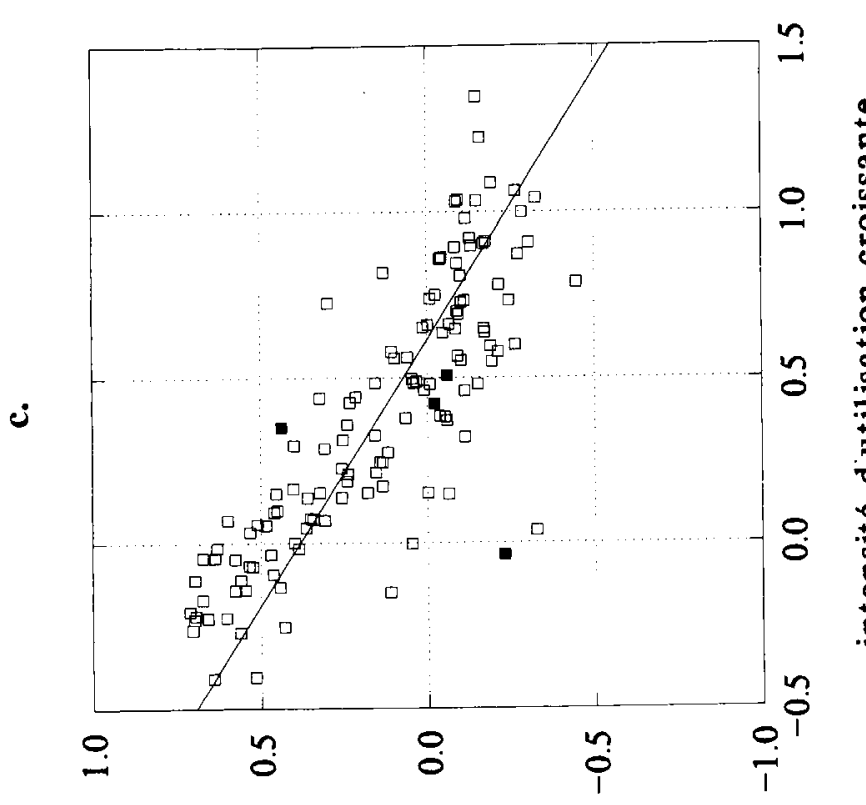

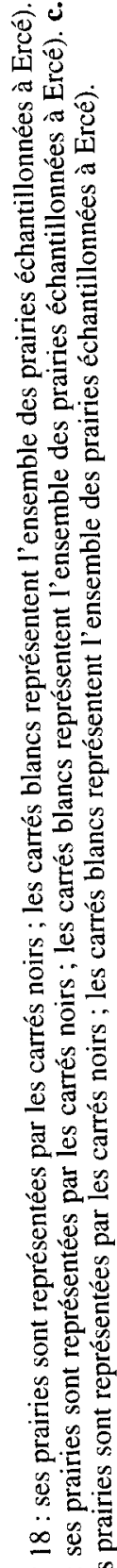

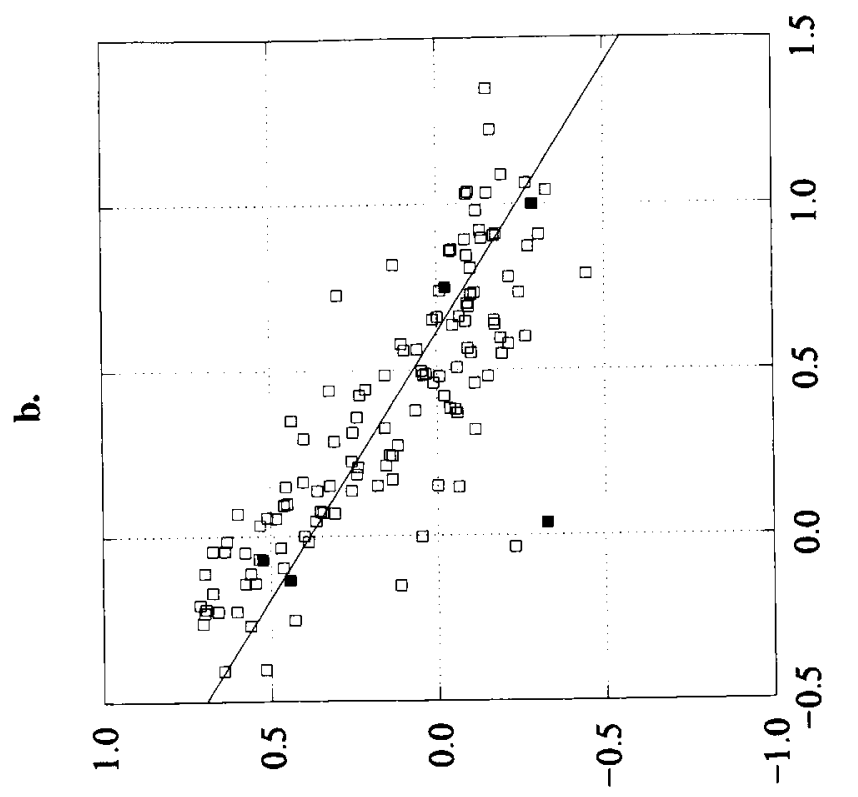

$\because$

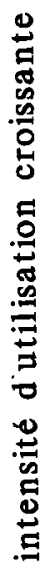

$\therefore \ddot{m}$

言

을

邓. 음

중응

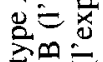

\%

흘

듕 $\overline{0}$

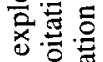

害运

$\rightarrow$ 중

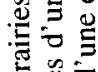

5.

$\frac{d y}{2 \pi}$ 


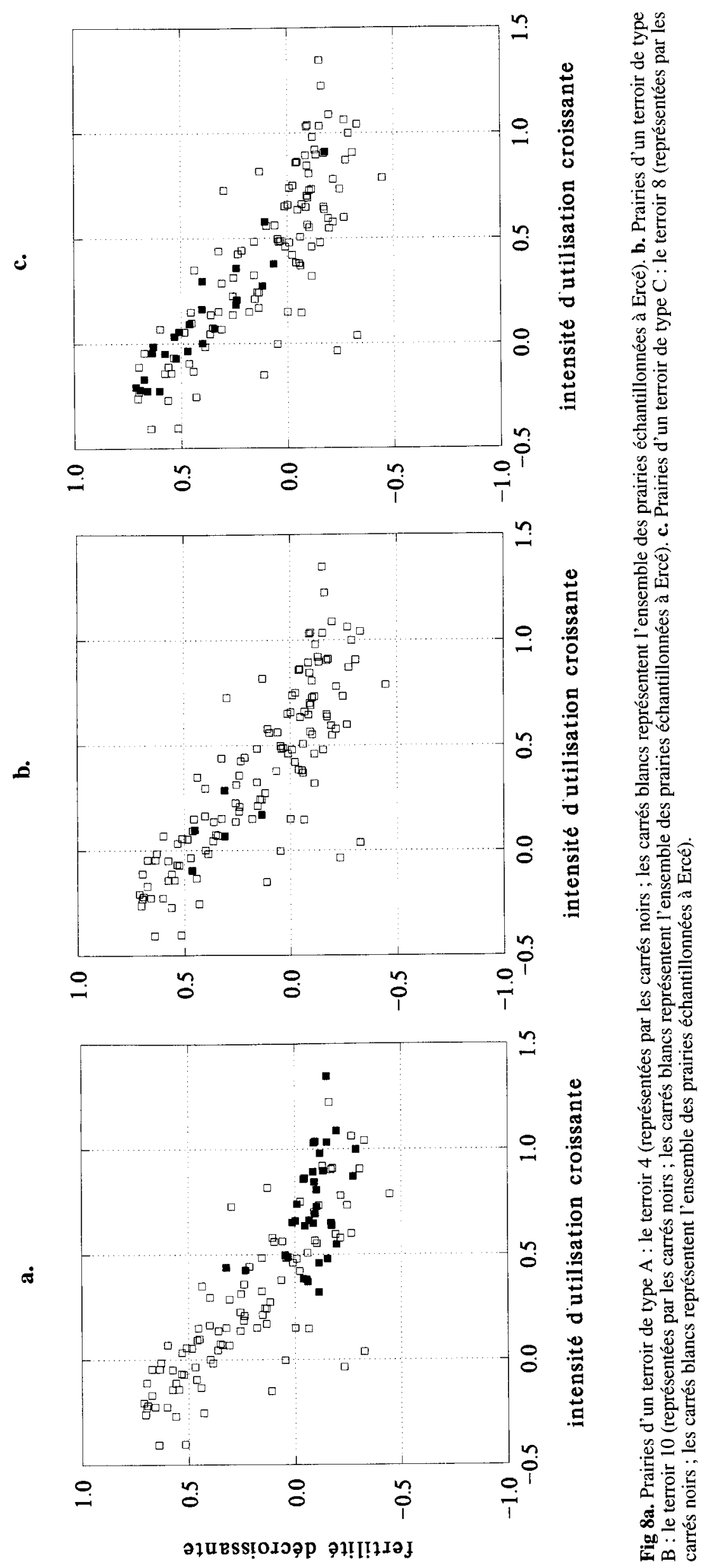



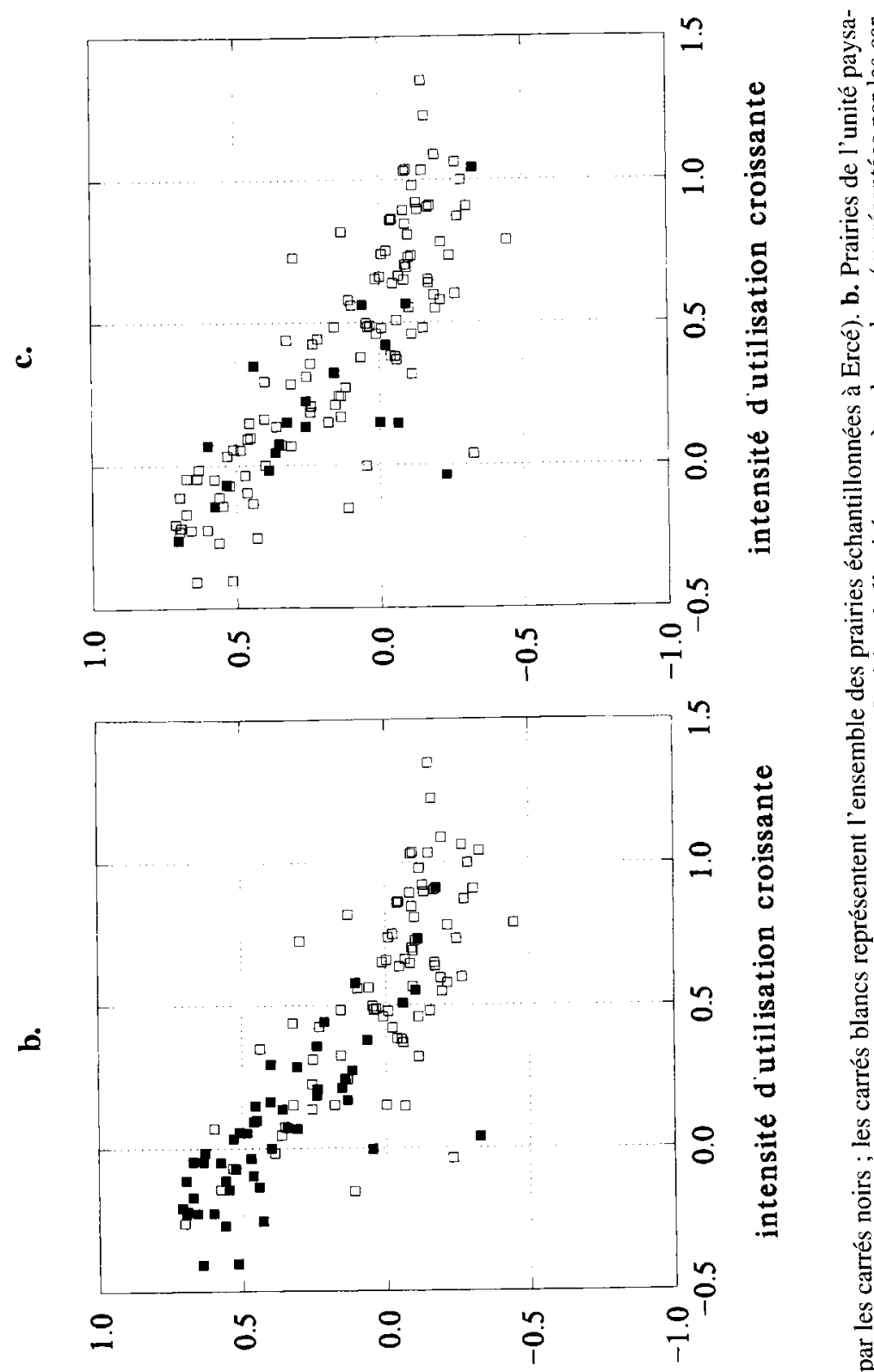

हैं 


\section{DISCUSSION}

\section{Hétérogénéité de la végétation au niveau des exploitations agricoles}

L'analyse de la végétation prairiale des exploitations montre une forte diversité dans la maîtrise des ressources pastorales. Cette diversité a été mise en relation avec des caractéristiques des exploitations relatives à trois tendances majeures de l'utilisation agricole du territoire de montagne.

i) Le nombre de terroirs utilisés par exploitation est un indicateur de la structure spatiale des exploitations, qui a tendance à se concentrer progressivement autour du siège de l'exploitation et sur les terroirs mécanisables (Gibon et al, 1995). Les exploitations agricoles d'Ercé utilisent un nombre de terroirs variant de 1 à 6 . Or les exploitations du groupe A (bonne maîtrise des ressources prairiales) utilisent un nombre élevé de terroirs (trois exploitations utilisent au moins quatre terroirs différents). L'utilisation d'un nombre élevé de terroirs est plus rare dans le groupe $B$ (maîtrise incomplète des ressources) : trois exploitations seulement sur six utilisent au moins quatre terroirs différents. Les exploitations du groupe $\mathrm{C}$, qui présentent un patron tout à fait éloigné de l'adéquation entre fertilité et intensité d'utilisation des prairies, utilisent un faible nombre de terroirs (deux et trois terroirs). La concentration des terres des exploitations sur un faible nombre de terroirs semble aller de pair avec la diminution de la maîtrise des ressources prairiales.

ii) La taille des exploitations en hectare est un indicateur de la tendance à l'agrandissement des exploitations agricoles. À Ercé elle varie entre 10 et 65 ha environ. Les exploitations du groupe A sont généralement de grandes exploitations (40-65 ha), tandis que les exploitations du groupe $B$, qui semblent perdre la maîtrise d'une partie de leurs ressources naturelles, sont petites $(<15 \mathrm{ha})$ à moyennes ( $<30 \mathrm{ha})$. L'agrandissement des exploitations agricoles semble jouer en faveur d'une gestion des ressources pastorales plus proche de leur fertilité.

iii) La pluriactivité des ménages agricoles est un indicateur de la diversification des activités des chefs d'exploitations. La plupart des exploitations du groupe B (maîtrise incomplète des ressources) sont conduites par des ménages pluriactifs (quatre exploitations), tandis que toutes les exploitations du groupe $\mathrm{C}$ (deux exploitations) appartiennent à des ménages pluriactifs. Ainsi la pluriactivité, qui est un facteur de maintien de l'activité agricole en zone de montagne, s'accompagne de la diminution de la maîtrise des ressources prairiales.

La relation entre chacune de ces trois variables, d'une part, et le degré de maîtrise des ressources, d'autre part, met en évidence une évolution contradictoire. Si la tendance à l'agrandissement des exploitations permet d'espérer une meilleure maîtrise des ressources, la tendance à la concentration du parcellaire des exploitations et à la diversification des activités des ménages s'accompagne d'une diminution de la maîtrise des ressources prairiales et soulève des inquiétudes sur l'avenir de pans entiers du territoire de montagne : les versants.

\section{Un modèle de représentation de l'évolution des stratégies des exploitations vis-à-vis des différents terroirs}

Ces différentes évolutions reflètent toutes la prédominance de stratégies individuelles des exploitations en matière de gestion de l'espace pastoral. Nous proposons une représentation de cette évolution, interprétée en termes de diversification des stratégies des éleveurs vis-à-vis des terroirs.

Si dans le cadre de l'ancienne agriculture de subsistance la répartition des exploitations dans les terroirs était homogène, chaque exploitation utilisant des parcelles sur chacun des différents terroirs (Chevalier, 1956), aujourd' hui cette répartition est beaucoup plus diversifiée. On passe d'une stratégie de coordination entre exploitations pour l'accès aux terroirs, basée sur la recherche d'une complémentarité des ressources au sein de chaque exploitation, à la mise en œuvre par les exploitants de trois stratégies différentes (fig 10):

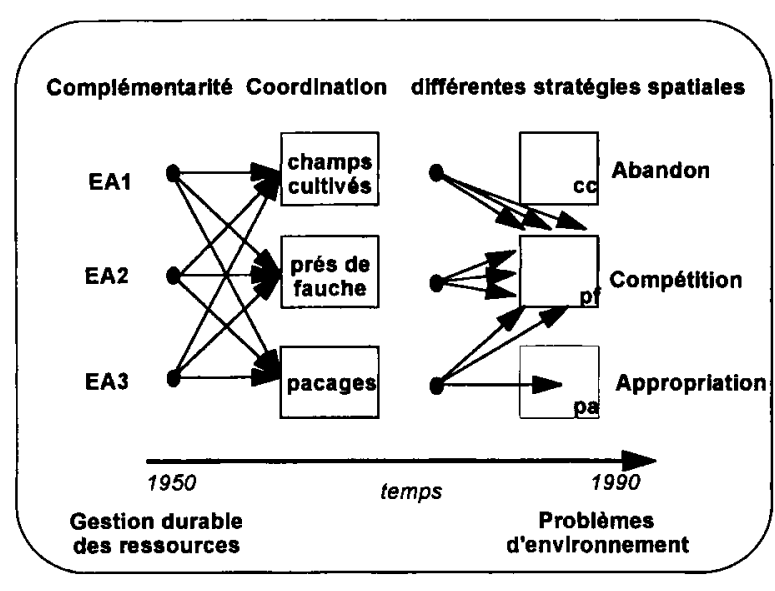

Fig 10. Représentation de l'évolution des stratégies d'accès aux terroirs par les exploitations agricoles (d'après Di Pietro, 1996). 
- compétition entre la plupart des exploitations pour l'accès aux ressources clés que constituent aujourd'hui les prés de fauche (terroir 4 , en fond de vallée, utilisé en prés de fauche par 13 exploitations ; terroir 8 , bordes proches du village, composé d'une mosaïque de parcelles de prés de fauche et de pacages, utilisé par 11 exploitations);

- appropriation de portions de territoire par un faible nombre d'exploitations (terroir 10, utilisé principalement en pacage par deux exploitations ; terroir 7, utilisé en prés de fauche par deux exploitations ; terroir 12, utilisé en prés de fauche par trois exploitations...);

- abandon de certains terroirs par les exploitations (terroir 14).

\section{Hétérogénéité de la végétation à l'échelle des terroirs}

À l'échelle des terroirs, nous observons des terroirs avec un type de végétation homogène (terroirs de fond de vallée - type $\mathrm{A}$ - ou bien terroirs de versant - type B) et des terroirs avec un type de végétation hétérogène (terroirs de versant : type C). Cette diversité a été mise en relation avec le nombre d'exploitations utilisatrices d'un terroir (nombre d'exploitations utilisant au moins une parcelle sur ce terroir).

Les terroirs de type $\mathrm{A}$ (végétation homogène en fond de vallée) sont utilisés par un nombre d'exploitations très variable; toutes mettent en œuvre sur ces terroirs mécanisables des pratiques intensives (deux, voire trois coupes, fertilisation régulière) et homogènes, qui expliquent l'homogénéité de la végétation.

Les terroirs de type $B$ (végétation homogène en versant) sont utilisés par un faible nombre d'exploitations : l'homogénéité de la végétation est expliquée par l'utilisation particulière du terroir de la part d'une exploitation, qui semble s'être appropriée un type de ressource pastorale et $\mathrm{y}$ avoir mis en œuvre des pratiques homogènes: pâturage d'été, fauche non intensive (une coupe)... C'est la manifestation sur le territoire de la stratégie d'appropriation décrite plus haut.

Les terroirs de type $C$ (végétation hétérogène en versant) sont utilisés par un nombre élevé d'exploitations, qui réagissent différemment aux contraintes que présentent ces terroirs (pente, accès difficile...), et y mettent eu œuvre des pratiques hétérogènes : utilisation en prés de fauche (une ou deux coupes...) ou utilisation exclusive en pacage (pâturage d'été ou de demi-saison...).
Ainsi, si la localisation des terroirs en fond de vallée explique leur utilisation intensive et leur végétation homogène, indépendamment du nombre d'exploitations utilisatrices, les versants sont le siège d'une grande diversité de pratiques et de types de végétation. Des terroirs utilisés par un nombre élevé d'exploitations, qui mettent en œuvre des pratiques diversifiées (végétation hétérogène, paysage en mosaïque), côtoient des terroirs à la végétation homogène, appropriés par une ou deux exploitations.

\section{Un modèle de représentation de l'évolution du paysage}

Les stratégies des exploitations vis-à-vis des ressources, présentées plus haut, se traduisent, sur le territoire, par un changement d'échelle de l'hétérogénéité du paysage. L'homogénéité des pratiques à l'échelle des terroirs avait autrefois engendré une partition du paysage en terroirs, caractérisés chacun par une fonction spécifique. Les terroirs représentaient donc le "grain» du paysage agraire, composant l'ensemble du territoire communal ou valléen ( «l'étendue », au sens d'Allen et al, 1984). Aujourd'hui l'hétérogénéité (des pratiques et de la végétation) intra-terroirs augmente, au dépens de l'hétérogénéité inter-terroirs. Les terroirs ne sont plus le grain adéquat pour appréhender l'utilisation du territoire pastoral, qui est plutôt représenté par la parcelle, seule portion d'espace avec des pratiques homogènes. Le territoire se présente comme une mosaïque de parcelles avec des pratiques et une végétation diversifiées (fig 11). Le maintien de cette hétérogénéité à l'échelle de la parcelle étant très coûteux en force de travail, la mosaïque fait progressivement place à une structure du paysage polarisée entre le fond de vallée et les versants, des unités paysagères à une échelle bien plus grossière que les terroirs.

Les deux modèles proposés (figs 10 et 11) présentent une comparaison entre un état de référence (le début de l'évolution) et un état observé (la situation actuelle). Ceci permet d'interpréter les situations observées actuellement comme différentes formes d'un seul processus (la transformation de l'utilisation des ressources par les exploitations), et de les replacer dans une trajectoire d'évolution. Ce choix méthodologique permet d'aller plus loin qu'un simple constat de diversité, et de formuler des scénarios d'évolution (van Andel et van den Bergh, 1987). 
Fig 11. Modélisation de l'évolution de la structure spatiale des paysages agraires (d'après Di Pietro, 1996). Dans les paysages pastoraux nous considérons l'hétérogénéité du paysage comme l'hétérogénéité des types de végétation priairiale.

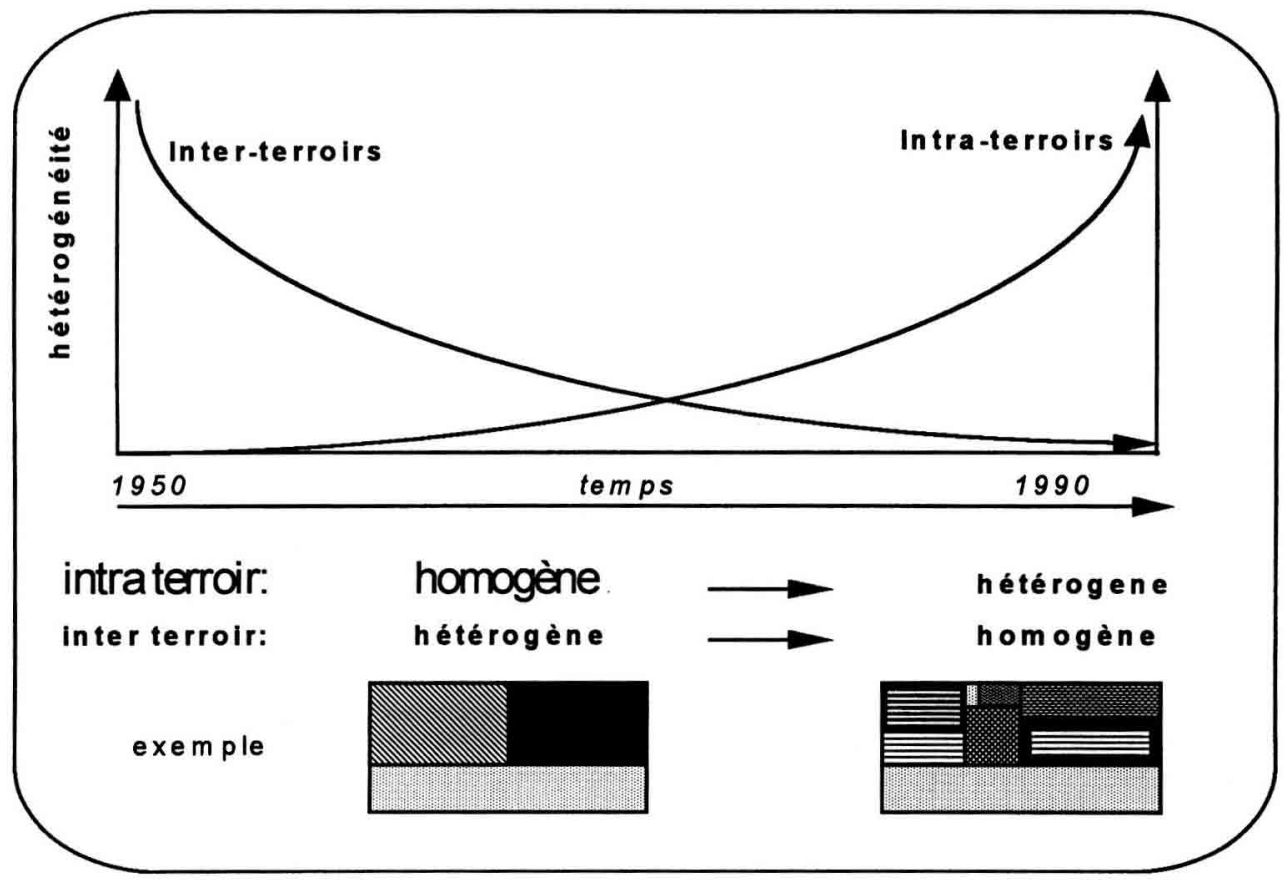

\section{Vers de nouveaux terroirs?}

À l'échelle des nouvelles unités paysagères, l'hétérogénéité de la végétation prairiale a été mise en relation avec la fonction agricole dominante. Aujourd'hui seul le fond de vallée a une fonction agricole spécifique: la fauche pour la constitution de stocks fourragers hivernaux. Cette homogénéité d'utilisation se traduit par une homogénéisation de la végétation, marquée par les pratiques agricoles liées à la fauche (régime de fertilisation et de prélèvements). Au contraire les deux unités de versant, les bordes et les soulanes, ne sont pas aujourd'hui caractérisées par une fonction agricole spécifique : elles sont utilisées pour partie comme pacages de demi-saison, pour partie comme estives privées, parfois elles sont encore fauchées. La dynamique de la végétation en leur sein est très diversifiée ; les prairies présentant une dynamique d'enfrichement sont situées justement sur ces unités paysagères de versant, notamment sur les soulanes.

L'abandon des versants préfigure donc une évolution ne laissant de place qu'à un seul type de ressource utilisée - les prés de fauche - et vers un paysage simplifié autour de deux entités :

- les prairies de fond de vallée, avec une fonction agricole marquée (constitution de stocks fourragers hivernaux par la fauche),
- les bois sur versant, dont la fonction reste à définir : il ne s'agit alors plus d'une fonction agricole mais d'une autre nature : sylvicole, cynégétique...

La gestion des ressources pastorales dans la commune d'Ercé, peu durable sur le plan écologique, est cependant performante sur le plan économique. En effet, elle correspond bien aux critères de viabilité économique dominants jusqu'à une époque récente : exploitations de grande taille, abandon des pratiques agricoles collectives... Dans d'autres sites de la même région, un paysage plus durable sur le plan écologique est géré par des exploitations moins viables sur le plan strictement économique (Di Pietro, 1996). Dans le contexte actuel de crise de l'agriculture, les politiques agricoles et d'aménagement du territoire peuvent jouer un rôle important pour sortir de cette dichotomie entre durabilité écologique et viabilité économique. Une approche pluri-échelle de la durabilité de la gestion agricole du territoire, comme celle que nous avons présenté ici, est alors nécessaire, comme le soulignent également Fresco et Kroonenberg (1992). Les mesures agri-environnementales devraient en effet s'appuyer sur des échelles adéquates, c'est-à-dire compatibles avec les niveaux d'organisation visés : c'est une condition importante du succès d'une politique durable de préservation des paysages. 


\section{REMERCIEMENTS}

Nous remercions J Seconds pour le premier dépouillement des données, qu'il a réalisé dans le cadre d'un mémoire d'ingénieur agronome. Nous remercions également D Magda, P Caniot, J Morère, pour la réalisation des relevés floristiques ainsi qu'A Gibon et JP Theau, pour leur participation à la conception et à la réalisation des enquêtes en exploitation. Merci à $\mathrm{C}$ Brau Nogué, C Icarán, et aux lecteurs anonymes désignés par la revue, pour une lecture attentive du manuscrit. $\mathrm{Ce}$ travail a été réalisé avec le soutien financier de la CEE (Contrat $n^{\circ} 90-0002$ ) et de l'Inra (direction des relations internationales et contrat Agrotech-Agreste «Pratiques d'élevage extensif »).

\section{RÉFÉRENCES}

Allen TFH (1987) Hierarchical complexity in ecology: a noneuclidean conception of the data space. Vegetatio 69, 17-25

Allen TFH, O'Neill RV, Hoekstra TW (1984) Interlevel relations in ecological research and management: some working principles from hierarchy theory. J Applied Systems Analysis 14, 63-79

Allen TFH, Starr TB (1982) Hierarchy. Perspectives for Ecological Complexity. The University of Chicago Press, Chicago, IL, États-Unis

Balent G (1986) The influence of grazing on the evolution of botanical composition of previously cultivated fields. The example of the Pyrenees. In : Rangeland: a Ressource under Siege (PJ Joss, PW Lynch, OB Williams, eds), Australian Academy of Science, 28-29

Balent G (1989) Hierarchical analysis of spatial patterns in pastoral systems. Etudes et Recherches sur les Systèmes Agraires et le Développement 16, $187-$ 198

Balent G (1991) Construction of a reference frame for studying the changes in species composition in grassland. Options Méditerranéennes 15, 73-81

Balent G, Duru M (1984) Influence des modes d'exploitation sur les caractéristiques et l'évolution des surfaces pastorales: cas des Pyrénées centrales. agronomie 4, 113-124

Balent G, Gibon A (1988) Définition et représentation du système pastoral. Niveaux d'organisation et pratiques de pâturage. Études et Recherches sur les Systèmes Agraires et le Développement 11, 65-78

Balent G, Stafford Smith M (1993) Conceptual model for evaluating the consequences of management practices on the use of pastoral resources. Proc 4th International Rangeland Congress, 22-27 avril 1991, Montpellier, 1158-1164
Baudry J (1990) Les problèmes d'échelle dans l'étude des changements globaux en écologie, $4^{\mathrm{e}}$ Séminaire informatique de l'Orstom « Le transfert d'échelle », septembre 1990, Brest, 225-234

Bertrand G (1975) Pour une histoire écologique de la France rurale. In : Histoire de la France rurale (G Duby, A Wallon, eds), tome 1, Paris, 37-111

Burel F, Baudry J, Clergeau P, Constant P, Etbert MC (1992) Approche spatiale des phénomènes écologiques : échelles et hiérarchie. Bull Ecol 23, 93-101

Chevalier M (1956) La vie humaine dans les Pyrénées ariégeoises. Résonances, Toulouse

Daget P, Poissonnet J (1971) Une méthode d'analyse phytologique des prairies. Critères d'application. Ann Agron 5-41

Deffontaines JP (1973) Analyse du paysage et étude régionale des systèmes de production agricole. Economie Rurale 98, 3-13

Deffontaines JP (1988) Réflexions sur les unités d'analyse. Relations entre taxonomies différentes. In : $D e$ la touffe d'herbe au paysage (B Hubert, N Girault, eds), Inra-Sad, Paris, 13-28

Delcros P (1992) Écologie du paysage et dynamique végétale post-culturale. Thèse de doctorat de l'université de Grenoble-I, Cemagref, 334 p

Di Pietro F (1996) Durabilité et organisation du paysage. Application des concepts de l'écologie systémique au diagnostic de la gestion pastorale du territoire des vallées des Pyrénées centrales (France). Thèse de doctorat de l'université de Toulouse-III, Inra, $262 \mathrm{p}+$ annexes

Fresco LO, Kroonenberg SB (1992) Time and spatial scales in ecological sustainability. Land Use Policy, juillet 1992, 155-168

Gibon A (1981) Pratiques d'éleveurs et résultats d'élevage dans les Pyrénées centrales. Thèse de docteuringenieur, Ina-Paris-Grigon, $106 \mathrm{p}$

Gibon A, Di Pietro F, Theau JP (1995) Stratégies d'utilisation de l'espace en montagne. I. Les structures spatiales des exploitations pyrénéennes. Cahiers Options Méditerranéennes 12, 183-196

Golley F (1989) A proper scale. Landscape Ecology 2, $71-72$

Inra-Enssaa (1977) Pays, paysans, paysages dans les Vosges du Sud. Inra, Paris

Meentemeyer V, Box EO (1987) Scale effects in landscape studies. In : Landscape Heterogeneity and Disturbance (MG Turner, ed), Springer-Verlag, New York, 15-36

Sauget N, Balent G (1993) The diversity of agricultural practices and landscape dynamics: the case of a hill region in the south-west of France. In : Landscape Ecology and Agroecosystems (RGH Bunce, L Ryszkowski, MG Paoletti, eds), Lewis Publishers, Boca Raton, 113-129

van Andel J, van den Bergh JP (1987) Outline of the theme. In : Disturbance in Grasslands (J van Andel, 
JP Bakker, RW Snaydon, eds), Dr Junk Publishers, Dordrecht, 3-13

Viviani Rossi E, Theau JP, Gibon A, Duru M (1992) Diagnostic des systèmes fourragers à partir d'une enquête : méthodologie et application à la constitu- tion des stocks fourragers dans le Couserans. Fourrages 130, 123-147

Wiens JA (1989) Spatial scaling in ecology. Functional Ecology 3, 385-399 